\title{
Bloch wave propagation in two-dimensional photonic crystals: Influence of the polarization
}

\author{
B. L O M B A R D E T*, L. A. D U N B A R, R. FER R I I A N D R. H O U R É \\ Institut de Photonique et d'Electronique Quantique, Ecole Polytechnique Fédérale de Lausanne, CH-1015 \\ Lausanne, Switzerland \\ (*author for correspondence: E-mail: benoit.lombardet@epfl.ch)
}

\begin{abstract}
Transverse Magnetic (TM) and Transverse Electric (TE) optical Bloch waves are the generic solutions of Maxwell's equations in two-dimensional photonic crystals (2D-PhCs). We present an intuitive description of these waves based on their Fourier decomposition into series of electromagnetic waves. The properties of these electromagnetic waves as well as their contribution to the global energy and group velocity of the global Bloch wave are discussed for each polarization. This description provides a simple and intuitive method to understand dispersion and group velocity effects in 2D-PhCs.
\end{abstract}

Key words: photonic crystal, Bloch wave, Fourier analysis, left-handed materials

\section{Introduction}

In the early $80 \mathrm{~s}$, the original refraction properties of periodic structures were demonstrated at optical wavelengths in thin film corrugated waveguides (Russell 1986a; Zengerle 1987). Similar effects such as negative refraction (Notomi 2000; Luo et al. 2002; Foteinopoulou et al. 2003; Cubukcu et al. 2003; Parimi et al. 2003), superprism (Kosaka et al. 1998; Baba and Nakamura 2002; Wu et al. 2002) and self-collimation (Kosaka et al. 1999; Witzens et al. 2002; Witzens and Scherer 2003) have recently been predicted and observed experimentally in two-dimensional photonic crystals (2D-PhCs). The physical understanding and use of such phenomena for designing new optical devices requires a thorough understanding of the basic properties of light propagation in periodic structures (Russell 1986b; Chu and Tamir 1970; Chu and Tamir 1972).

In this paper, we present a description of light propagation in 2D-PhCs based on the Fourier analysis of optical Bloch waves. This approach provides a clear understanding of the basic properties of Bloch waves in $\mathrm{PhCs}$ (e.g. wave vector, energy density, group velocity ...) and highlights some differences between the TE and TM polarizations. Moreover, this formalism provides a simple method to investigate the original dispersion properties of 2D-PhCs.

In Section 2, we apply the plane wave expansion (PWE) method to solve Maxwell's equations and calculate the Fourier transform of the magnetic, electric and displacement fields of an optical Bloch wave in a 2D-PhC. In 
Section 3, we use the different Fourier components of these fields to express the optical Bloch wave as a series of electromagnetic waves. Then we demonstrate that the latter waves possess the basic properties of electromagnetic plane waves. We then explain the contribution of each individual component to the global energy and group velocity and discuss the valid domain of this decomposition. In Section 4, we introduce a possible graphical representation of optical Bloch waves in reciprocal space and consider two examples to illustrate the insight of this description.

\section{Maxwell's equations in 2D-PhCs}

The 2D-PhC structure consists of a 2D lattice of dielectric cylinders with permittivity $\varepsilon_{2}$ embedded in a dielectric medium with permittivity $\varepsilon_{1}$. These materials are assumed to be non-magnetic and thus are assigned a magnetic permittivity equal to the vacuum magnetic permittivity $\mu_{0}$. The primitive lattice vectors are $\mathbf{a}_{1}$ and $\mathbf{a}_{2}$, the section of the cylinders has an arbitrary shape and the $z$-axis is chosen parallel to the cylinders, see Fig. 1.

\subsection{MAXWELL'S EQUATIONS IN REAL SPACE}

We consider the propagation of optical Bloch waves in the $x y$-plane of the $2 \mathrm{D}-\mathrm{PhC}$ described in Fig. 1. These waves are characterized by their wave vector $\mathbf{k}=\left(k_{x}, k_{y}, 0\right)$.

TE-polarized Bloch waves have a magnetic field parallel to the $z$-axis and electric and displacement fields perpendicular to the $z$-axis:

$$
\mathbf{H}_{\mathbf{k}}=\left(0,0, H_{z \mathbf{k}}\right), \quad \mathbf{E}_{\mathbf{k}}=\left(E_{x \mathbf{k}}, E_{y \mathbf{k}}, 0\right) \quad \text { and } \quad \mathbf{D}_{\mathbf{k}}=\left(D_{x \mathbf{k}}, D_{y \mathbf{k}}, 0\right) \text {. }
$$

TM-polarized Bloch waves have a magnetic field perpendicular to the $z$-axis and electric and displacement fields parallel to the $z$-axis:

$$
\mathbf{H}_{\mathbf{k}}=\left(H_{x \mathbf{k}}, H_{y \mathbf{k}}, 0\right), \quad \mathbf{E}_{\mathbf{k}}=\left(0,0, E_{z \mathbf{k}}\right) \text { and } \mathbf{D}_{\mathbf{k}}=\left(0,0, D_{z \mathbf{k}}\right) .
$$

In the real space, the components of these fields are linked together via Maxwell's equations:

$$
\begin{aligned}
& \nabla \cdot \mathbf{H}_{\mathbf{k}}=0, \\
& \nabla \cdot \mathbf{D}_{\mathbf{k}}=0, \\
& \nabla \times \mathbf{H}_{\mathbf{k}}=-i \omega \mathbf{D}_{\mathbf{k}}, \\
& \nabla \times \mathbf{E}_{\mathbf{k}}=i \omega \mu_{0} \mathbf{H}_{\mathbf{k}},
\end{aligned}
$$




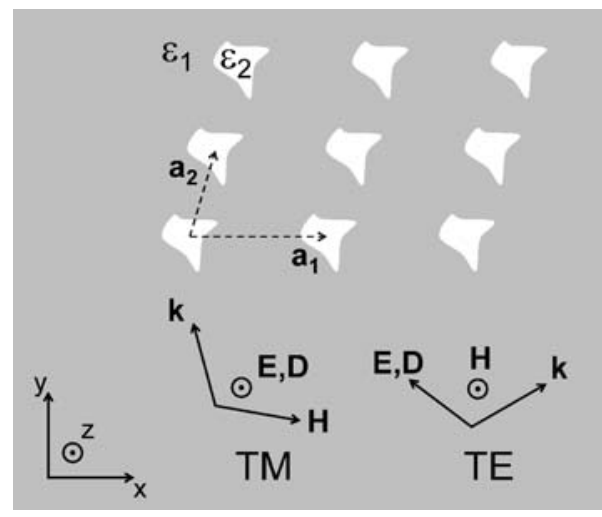

Fig. 1. Top view of the investigated 2D-PhC. A $2 \mathrm{D}$ lattice of dielectric cylinders with permittivity $\varepsilon_{2}$ is embedded in a dielectric medium with permittivity $\varepsilon_{1}$. The section of the cylinder has an arbitrary shape. The elementary lattice vectors are $\mathbf{a}_{1}$ and $\mathbf{a}_{2}$. We consider TM-polarized $\left(\mathbf{E} \| \mathbf{e}_{\mathbf{z}}\right)$ and TE-polarized $\left(\mathbf{H} \| \mathbf{e}_{\mathbf{z}}\right)$ Bloch waves propagating in the $x y$-plane perpendicular to the cylinders.

and with the constitutive relation:

$$
\mathbf{D}_{\mathbf{k}}=\varepsilon_{0} \varepsilon \mathbf{E}_{\mathbf{k}}
$$

where the permittivity $\varepsilon=\varepsilon_{(\mathbf{r})}$ is a scalar function with the same periodicity as the $2 \mathrm{D}$ lattice.

\subsection{FOURIER DECOMPOSITION OF $\mathbf{H}_{\mathbf{k}}, \mathbf{E}_{\mathbf{k}}$ AND $\mathbf{D}_{\mathbf{k}}$}

According to Bloch's theorem (Sakoda 2001), each field $\mathbf{A}_{\mathbf{k}}(\mathbf{r})(\mathbf{A}=\mathbf{H}, \mathbf{E}$ or D) can be written as a Bloch wave:

$$
\mathbf{A}_{\mathbf{k}}(\mathbf{r})=\exp (\mathbf{i k} \cdot \mathbf{r}) \mathcal{A}_{\mathbf{k}}(\mathbf{r})
$$

where $\mathbf{k} \in\left[-\frac{\pi}{a}, \frac{\pi}{a}\left[\times\left[-\frac{\pi}{a}, \frac{\pi}{a}\left[\right.\right.\right.\right.$ is the wave vector and $\mathcal{A}_{\mathbf{k}}(\mathbf{r})$ is a vectorial function with the same periodicity as the $2 \mathrm{D}$ lattice. The corresponding reciprocal lattice can be described by the series of reciprocal vectors $\mathbf{G}_{n, m}=n \mathbf{G}_{1}+m \mathbf{G}_{2}$, where $\mathbf{G}_{1}=2 \pi\left(\mathbf{a}_{\mathbf{2}} \times \mathbf{e}_{\mathbf{z}}\right) / S$ and $\mathbf{G}_{2}=2 \pi\left(\mathbf{e}_{\mathbf{z}} \times \mathbf{a}_{\mathbf{1}}\right) / S$ with $S=\mathbf{a}_{\mathbf{1}} \cdot\left(\mathbf{a}_{\mathbf{2}} \times \mathbf{e}_{\mathbf{z}}\right)$. Due to the periodicity, $\mathbf{A}_{\mathbf{k}}$ can be expanded as a Fourier series:

$$
\mathbf{A}_{\mathbf{k}}(\mathbf{r})=\sum_{n, m} \mathcal{A}_{n, m(\mathbf{k})} \exp \left(\mathrm{i}\left(\mathbf{k}+\mathbf{G}_{n, m}\right) \cdot \mathbf{r}\right)
$$

where the $\mathbf{G}_{n, m}$ are the vectors of the reciprocal lattice and the $\mathcal{A}_{n, m(\mathbf{k})}$ are the Fourier components of the vectorial function $\mathcal{A}_{\mathbf{k}}$. 
The Fourier component $\mathcal{H}_{n, m(\mathbf{k})}$ of the magnetic field can be expressed as $\mathcal{H}_{n, m(\mathbf{k})}=H_{0} \mathbf{h}_{n, m(\mathbf{k})}$, where $H_{0}$ is the amplitude of the total magnetic field and $\mathbf{h}_{n, m(\mathbf{k})}$ is a dimensionless vector. Thus, the $\mathbf{h}_{n, m(\mathbf{k})}$ satisfy the normalization relation $\sum_{n, m}\left|\mathbf{h}_{n, m(\mathbf{k})}\right|^{2}=1$.

In order to manipulate dimensionless quantities and to simplify the following calculations, the Fourier components $\mathcal{E}_{n, m(\mathbf{k})}$ and $\mathcal{D}_{n, m(\mathbf{k})}$ can be expressed without loss of generality as $\mathcal{E}_{n, m(\mathbf{k})}=\mu_{0} c H_{0} \mathbf{e}_{n, m(\mathbf{k})}$ and $\mathcal{D}_{n, m(\mathbf{k})}=\left(H_{0} / c\right) \mathbf{d}_{n, m(\mathbf{k})}$, where $c$ is the speed of light in vacuum and $\mathbf{e}_{n, m(\mathbf{k})}$ and $\mathbf{d}_{n, m(\mathbf{k})}$ are dimensionless vectors.

Finally, the magnetic, electric and displacement fields of the Bloch wave take the form:

$$
\begin{aligned}
& \mathbf{H}_{\mathbf{k}}(\mathbf{r})=\sum_{n, m} \mathcal{H}_{n, m(\mathbf{k})} \exp \left(\mathrm{ik}_{n, m} \cdot \mathbf{r}\right)=\sum_{n, m} H_{0} \mathbf{h}_{n, m(\mathbf{k})} \exp \left(\mathrm{ik}_{n, m} \cdot \mathbf{r}\right), \\
& \mathbf{E}_{\mathbf{k}}(\mathbf{r})=\sum_{n, m} \mathcal{E}_{n, m(\mathbf{k})} \exp \left(\mathrm{ik}_{n, m} \cdot \mathbf{r}\right)=\sum_{n, m} \mu_{0} c H_{0} \mathbf{e}_{n, m(\mathbf{k})} \exp \left(\mathrm{ik}_{n, m} \cdot \mathbf{r}\right), \\
& \mathbf{D}_{\mathbf{k}}(\mathbf{r})=\sum_{n, m} \mathcal{D}_{n, m(\mathbf{k})} \exp \left(\mathrm{ik}_{n, m} \cdot \mathbf{r}\right)=\sum_{n, m} \frac{H_{0}}{c} \mathbf{d}_{n, m(\mathbf{k})} \exp \left(\mathrm{i} \mathbf{k}_{n, m} \cdot \mathbf{r}\right),
\end{aligned}
$$

where $\mathbf{k}_{n, m}=\mathbf{k}+\mathbf{G}_{n, m}$.

Equations (10-12) are valid for both polarizations and differ only in the orientation of the dimensionless vectors $\mathbf{h}_{n, m(\mathbf{k})}, \mathbf{e}_{n, m(\mathbf{k})}$ and $\mathbf{d}_{n, m(\mathbf{k})}$.

\subsection{MAXWELL'S EQUATIONS IN RECIPROCAL SPACE}

Inserting Equations (10-12) into Maxwell's Equations (3-6) and the constitutive relation Equation (7), we obtain the following relations between the Fourier components of the different fields:

$$
\begin{aligned}
& \mathbf{k}_{n, m} \cdot \mathbf{h}_{n, m(\mathbf{k})}=0 \\
& \mathbf{k}_{n, m} \cdot \mathbf{d}_{n, m(\mathbf{k})}=0 \\
& \mathbf{d}_{n, m(\mathbf{k})}=-\frac{\mathbf{k}_{n, m} c}{\omega} \times \mathbf{h}_{n, m(\mathbf{k})}, \\
& \mathbf{h}_{n, m(\mathbf{k})}=\frac{\mathbf{k}_{n, m} c}{\omega} \times \mathbf{e}_{n, m(\mathbf{k})}, \\
& \mathbf{e}_{n, m(\mathbf{k})}=\sum_{n^{\prime}, m^{\prime}} \kappa_{n-n^{\prime}, m-m^{\prime}} \mathbf{d}_{n^{\prime}, m^{\prime}(\mathbf{k})},
\end{aligned}
$$

where the $\kappa_{n, m}$ are the Fourier coefficients of the periodic function $1 / \varepsilon_{(\mathbf{r})}=\sum_{n, m} \kappa_{n, m} \exp \left(\mathrm{iG}_{n, m} \cdot \mathbf{r}\right)$. 
TM polarization solution. In this case, $\mathbf{e}_{n, m(\mathbf{k})}=e_{n, m(\mathbf{k}) z} \mathbf{e}_{\mathbf{z}}$ and $\mathbf{d}_{n, m(\mathbf{k})}=d_{n, m(\mathbf{k}) z} \mathbf{e}_{\mathbf{z}}$. Substituting Equations (15) and (16) into Equation (17) gives the equation for the coefficients $e_{n, m(\mathbf{k}) z}$ :

$$
\sum_{n^{\prime}, m^{\prime}} \kappa_{n-n^{\prime}, m-m^{\prime}}\left|\mathbf{k}_{n, m}\right|\left|\mathbf{k}_{n^{\prime}, m^{\prime}}\right| \frac{e_{n^{\prime}, m^{\prime}(\mathbf{k}) z}}{\left|\mathbf{k}_{n, m}\right|}=\left(\frac{\omega}{c}\right)^{2} \frac{e_{n, m(\mathbf{k}) z}}{\left|\mathbf{k}_{n, m}\right|}
$$

For an arbitrarily large integer $N$, the truncated version of Equation (18) with $|n|,|m| \leq N$ is the standard eigenvalues equation used in the PWE method to compute the dispersion relations of TM-polarized waves in 2D-PhCs (P1ihal and Maradudin 1991). The eigenvectors give the coefficients $e_{n, m(\mathbf{k})} /\left|\mathbf{k}_{n, m}\right|$ from which the vectors $\mathbf{e}_{n, m(\mathbf{k})}$ are easily deduced. The vectors $\mathbf{h}_{n, m(\mathbf{k})}$ and $\mathbf{d}_{n, m(\mathbf{k})}$ can then be calculated from Equations (15) and (16).

$T E$ polarization solution. In this case, $\mathbf{h}_{n, m(\mathbf{k})}=h_{n, m(\mathbf{k}) z} \mathbf{e}_{\mathbf{z}}$. Substituting Equations (15) and (17) into Equation (16) gives the equation for the coefficients $h_{n, m(\mathbf{k}) z}$ :

$$
\sum_{n^{\prime}, m^{\prime}} \kappa_{n-n^{\prime}, m-m^{\prime}}\left(\mathbf{k}_{n, m} \cdot \mathbf{k}_{n^{\prime}, m^{\prime}}\right) h_{n^{\prime}, m^{\prime}(\mathbf{k}) z}=\left(\frac{\omega}{c}\right)^{2} h_{n, m(\mathbf{k}) z}
$$

As in the TM case, the truncated version of Equation (19) with $|n|,|m| \leq N$ is an eigenvalues equation whose eigenvectors give the vectors $\mathbf{h}_{n, m(\mathbf{k})}$ from which the vectors $\mathbf{d}_{n, m(\mathbf{k})}$ and $\mathbf{e}_{n, m(\mathbf{k})}$ are easily deduced using Equation (15) and (17).

\section{Fourier analysis of the optical Bloch wave}

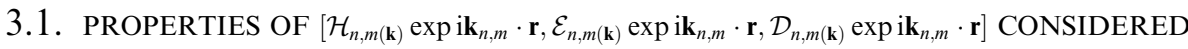 AS AN ELECTROMAGNETIC WAVE}

We consider the electromagnetic wave $\mathcal{F}_{n, m(\mathbf{k})}$ built arbitrarily with the magnetic, electric and displacement fields of the $(n, m)^{\text {th }}$ Fourier components of the global Bloch wave. This wave is characterized by the wave vector $\mathbf{k}_{n, m}$, the magnetic field $\mathcal{H}_{n, m(\mathbf{k})} \exp \left(\mathrm{ik}_{n, m} \cdot \mathbf{r}\right)=H_{0} \mathbf{h}_{n, m(\mathbf{k})} \exp \left(\mathrm{ik}_{n, m} \cdot \mathbf{r}\right)$, the electric field $\mathcal{E}_{n, m(\mathbf{k})} \exp \left(\mathrm{ik}_{n, m} \cdot \mathbf{r}\right)=\mu_{0} c H_{0} \mathbf{e}_{n, m(\mathbf{k})} \exp \left(i \mathbf{k}_{n, m} \cdot \mathbf{r}\right)$ and the displacement field $\mathcal{D}_{n, m}(\mathbf{k}) \exp \left(\mathrm{ik}_{n, m} \cdot \mathbf{r}\right)=\left(H_{0} / c\right) \mathbf{d}_{n, m(\mathbf{k})} \exp \left(\mathbf{i k}_{n, m} \cdot \mathbf{r}\right)$.

In order to compare $\mathcal{F}_{n, m(\mathbf{k})}$ with an electromagnetic plane wave with wave vector $\mathbf{k}_{n, m}$, two points must be considered. First, $\mathcal{F}_{n, m(\mathbf{k})}$ must satisfy Maxwell's equations: from Equations (13-16), it is clear that Equations (3-6) are satisfied. Second, the constitutive relation between $\mathcal{E}_{n, m(\mathbf{k})}$ and $\mathcal{D}_{n, m(\mathbf{k})}$ must be clarified. There is a difference between the TM and TE polarizations as can be seen by their representation in Fig. 2a and b, respectively. 
TM polarization. Using Equations (15) and (16) and the relation $\mathbf{k}_{n, m} \cdot \mathbf{e}_{n, m(\mathbf{k})}=0$ which is specific to this polarization, we obtain:

$$
\mathbf{d}_{n, m(\mathbf{k})}=-\frac{\mathbf{k}_{n, m} c}{\omega} \times\left(\frac{\mathbf{k}_{n, m} c^{2}}{\omega} \times \mathbf{e}_{n, m(\mathbf{k})}\right)=\left|\frac{\mathbf{k}_{n, m} c}{\omega}\right|^{2} \mathbf{e}_{n, m(\mathbf{k})}
$$

We conclude that the TM electromagnetic wave $\mathcal{F}_{n, m(\mathbf{k})}$ behaves like a TM electromagnetic plane wave propagating with energy $\omega$ and wave vector $\mathbf{k}_{n, m}$ in a homogeneous medium with the permittivity $\varepsilon_{0}\left|\mathbf{k}_{n, m} c / \omega\right|^{2}$. Nevertheless, we would like to stress that Equation (17) connects the different $\mathcal{F}_{n, m(\mathbf{k})}$ together and imposes their relative amplitudes through its equivalent form Equation (18). Thus, the waves $\mathcal{F}_{n, m(\mathbf{k})}$ cannot exist independently, but only as components of the total Bloch wave. Because of this peculiarity, the $\mathcal{F}_{n, m(\mathbf{k})}$ cannot be named electromagnetic plane waves, so we will call them partial electromagnetic plane waves in following sections.

$T E$ polarization. Contrary to the TM case, the vectors $\mathbf{d}_{n, m(\mathbf{k})}$ of the different $\mathcal{F}_{n, m(\mathbf{k})}$ are not parallel and reside in the $x y$-plane, so that Equation (17) is now a vectorial equation in the $x y$-plane, see Fig. $2 \mathrm{~b}$. The compatibility of Equation (17) with Maxwell's equations yields Equation (19) and imposes the relative amplitudes of the different $\mathcal{F}_{n^{\prime}, m^{\prime}(\mathbf{k})}$, and consequently the $\mathbf{e}_{n^{\prime}, m^{\prime}(\mathbf{k})}$, see Section 2.3.

Thus, the vectors $\mathcal{E}_{n, m(\mathbf{k})}$ and $\mathcal{D}_{n, m(\mathbf{k})}$ of the TE wave $\mathcal{F}_{n, m(\mathbf{k})}$ are not necessarily parallel. The same effect occurs in anisotropic media where the permittivity $\varepsilon$ is a tensor. Here, according to Equation (17), the electric field $\mathcal{E}_{n, m(\mathbf{k})}$ is not related by an hermitian operator to the displacement field $\mathcal{D}_{n, m(\mathbf{k})}$ but is a linear combination of the displacement field of all the different $\mathcal{F}_{n^{\prime}, m^{\prime}(\mathbf{k})}$.
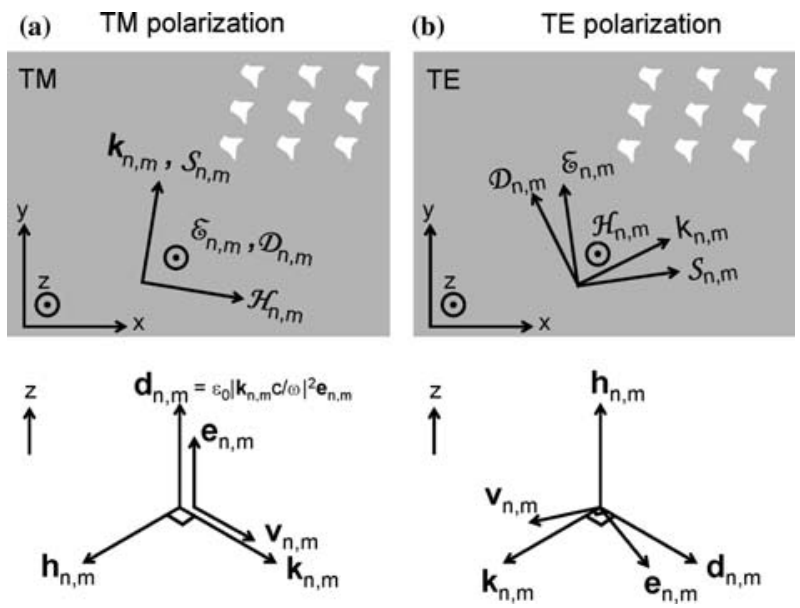

Fig. 2. Graphical representation of the electromagnetic wave $\mathcal{F}_{n, m(\mathbf{k})}$ with magnetic field $\mathcal{H}_{n, m(\mathbf{k})}$, electric field $\mathcal{E}_{n, m(\mathbf{k})}$ and displacement field $\mathcal{D}_{n, m(\mathbf{k})}$ (a) TM polarization (b) TE polarization. 
Naming $\mathbf{e}_{n, m(\mathbf{k}) \|}$ and $\mathbf{e}_{n, m(\mathbf{k}) \perp}$ the parallel and perpendicular component of $\mathbf{e}_{n, m(\mathbf{k})}$ along the direction of $\mathbf{k}_{n, m}$, we consider the electromagnetic wave $\widetilde{\mathcal{F}}_{n, m(\mathbf{k})}$ built arbitrarily with the magnetic $\left(\mathcal{H}_{n, m(\mathbf{k})}\right)$, orthogonal electric $\left(\mathcal{E}_{n, m(\mathbf{k}) \perp}\right)$ and displacement $\left(\mathcal{D}_{n, m(\mathbf{k})}\right)$ Fourier components.

$$
\mathbf{d}_{n, m(\mathbf{k})}=-\frac{\mathbf{k}_{n, m} c}{\omega} \times\left(\frac{\mathbf{k}_{n, m} c}{\omega} \times \mathbf{e}_{n, m(\mathbf{k})}\right)=\left|\frac{\mathbf{k}_{n, m} c}{\omega}\right|^{2} \mathbf{e}_{n, m(\mathbf{k}) \perp} .
$$

Hence, the electromagnetic wave $\widetilde{\mathcal{F}}_{n, m(\mathbf{k})}$ behaves like a TE electromagnetic plane wave propagating with energy $\omega$ and wave vector $\mathbf{k}_{n, m}$ in an homogeneous medium with the permittivity $\varepsilon_{0}\left|\mathbf{k}_{n, m} c / \omega\right|^{2}$.

The TE wave $\mathcal{F}_{n, m(\mathbf{k})}$ which also satisfies Maxwell's equations is identical to $\widetilde{\mathcal{F}}_{n, m(\mathbf{k})}$ except that its electric field possess an extra-component along the direction of $\mathbf{k}_{n, m}$. The influence of this extra-component will be discussed below.

As for TM polarization, the waves $\mathcal{F}_{n, m(\mathbf{k})}$ only exist as components of the whole Bloch wave, so we will also call them partial electromagnetic plane waves in following sections.

\subsection{ENERGY PROPAGATION OF THE BLOCH WAVE}

\subsubsection{Time-space averaged energy density}

Using Parseval-Plancherel equality, we calculate the time-space average magnetic and electric energy densities $\left\langle\mathbb{E}_{\text {magn. }(\mathbf{k})}\right\rangle_{t, s}$ and $\left\langle\mathbb{E}_{\text {elec.(k) }}\right\rangle_{t, s}$ of the global Bloch wave:

$$
\begin{aligned}
\left\langle\mathbb{E}_{\text {magn. }(\mathbf{k})}\right\rangle_{t, s} & =\left\langle\frac{1}{2} \mu_{0} \frac{\mathbf{H} \cdot \mathbf{H}^{*}}{2}\right\rangle_{s}=\sum_{n, m} \frac{1}{4} \mu_{0} \mathcal{H}_{n, m(\mathbf{k})} \cdot \mathcal{H}_{n, m(\mathbf{k})}^{*} \\
& =\sum_{n, m} \frac{1}{4} \mu_{0}\left|\mathbf{h}_{n, m(\mathbf{k})}\right|^{2} H_{0}^{2} \\
\left\langle\mathbb{E}_{\text {elec. }(\mathbf{k})}\right\rangle_{t, s} & =\left\langle\frac{1}{2} \frac{\mathbf{E} \cdot \mathbf{D}^{*}}{2}\right\rangle_{s}=\sum_{n, m} \frac{1}{4} \mathcal{E}_{n, m(\mathbf{k})} \cdot \mathcal{D}_{n, m(\mathbf{k})}^{*} \\
& =\sum_{n} \frac{1}{4} \mu_{0} H_{0}^{2} \mathbf{e}_{n, m(\mathbf{k})} \cdot\left(\mathbf{h}_{n, m(\mathbf{k})}^{*} \times \frac{\mathbf{k}_{n, m} c}{\omega}\right) \\
& =\sum_{n} \frac{1}{4} \mu_{0}\left|\mathbf{h}_{n, m(\mathbf{k})}\right|^{2} H_{0}^{2}=\left\langle\mathbb{E}_{\operatorname{magn} .(\mathbf{k})}\right\rangle_{t, s} .
\end{aligned}
$$


The total time-space average energy density $\left\langle\mathbb{E}_{\mathbf{k}}\right\rangle_{t, s}$ of the global Bloch wave can be simply written as:

$$
\left\langle\mathbb{E}_{\mathbf{k}}\right\rangle_{t, s}=\sum_{n, m} \frac{1}{2} \mu_{0} \mathcal{H}_{n, m(\mathbf{k})} \cdot \mathcal{H}_{n, m(\mathbf{k})}^{*}=\sum_{n, m} \mathbb{E}_{n, m(\mathbf{k})}
$$

This expression is, of course, equal to $\frac{1}{2} \mu_{0} H_{0}^{2}$. Yet, the form above shows that the energy density of the global Bloch wave can be decomposed into fractional energy densities $\mathbb{E}_{n, m(\mathbf{k})}=\frac{1}{2} \mu_{0}\left|\mathbf{h}_{n, m(\mathbf{k})}\right|^{2} H_{0}^{2}$ corresponding to the energy density of the partial electromagnetic plane wave $\mathcal{F}_{n, m(\mathbf{k})}$ described in Section 3.1. This interesting result points out the importance of the dimensionless coefficients $\left|\mathbf{h}_{n, m(\mathbf{k})}\right|^{2}$ which give the relative energetic contributions of the different $\mathcal{F}_{n, m(\mathbf{k})}$. Finally it is important to note that this result is valid for both TM and TE polarizations.

\subsubsection{Time-space averaged Poynting vector}

Using the Parseval-Plancherel equality, we develop the time-space average Poynting vector $\left\langle\mathbf{S}_{\mathbf{k}}\right\rangle_{t, s}$ of the global Bloch wave:

$$
\begin{aligned}
\left\langle\mathbf{S}_{\mathbf{k}}\right\rangle_{t, s} & =\left\langle\Re e\left(\frac{\mathbf{E} \times \mathbf{H}^{*}}{2}\right)\right\rangle_{s}=\sum_{n, m} \Re e\left(\frac{1}{2} \mathcal{E}_{n, m(\mathbf{k})} \times \mathcal{H}_{n, m(\mathbf{k})}^{*}\right) \\
& =\sum_{n, m} \mathcal{S}_{n, m(\mathbf{k})} .
\end{aligned}
$$

The partial Poynting vector $\mathcal{S}_{n, m(\mathbf{k})}=\Re e\left(\frac{1}{2} \mathcal{E}_{n, m(\mathbf{k})} \times \mathcal{H}_{n, m(\mathbf{k})}^{*}\right)$ corresponds to the Poynting vector of the partial electromagnetic plane wave $\mathcal{F}_{n, m(\mathbf{k})}$, see Fig. 2.

$T M$ polarization. Using Equation (15) and the relation $\mathbf{k}_{n, m} \cdot \mathcal{E}_{n, m(\mathbf{k})}=0$ which is specific to the TM polarization, we obtain:

$$
\mathcal{S}_{n, m(\mathbf{k})}=\mathbb{E}_{n, m(\mathbf{k})} \frac{\omega}{\left|\mathbf{k}_{n, m}\right|^{2}} \mathbf{k}_{n, m}
$$

$T E$ polarization. Using the parallel and perpendicular components of $\mathcal{E}_{n, m(\mathbf{k})}$ defined in Section 3.1, $\mathcal{S}_{n, m(\mathbf{k})}$ can be decomposed as:

$$
\mathcal{S}_{n, m(\mathbf{k})}=\mathcal{S}_{n, m(\mathbf{k}) \|}+\mathcal{S}_{n, m(\mathbf{k}) \perp}
$$

where 


$$
\mathcal{S}_{n, m(\mathbf{k}) \|}=\mathbb{E}_{n, m(\mathbf{k})} \frac{\omega}{\left|\mathbf{k}_{n, m}\right|^{2}} \mathbf{k}_{n, m}
$$

and

$$
\mathcal{S}_{n, m(\mathbf{k}) \perp}=\Re e\left[\frac{1}{2}\left(\frac{\omega}{\left|\mathbf{k}_{n, m}\right|^{2}} \mathbf{k}_{n, m} \cdot \mathcal{E}_{n, m(\mathbf{k}) \|}\right) \mathcal{D}_{n, m(\mathbf{k})}^{*}\right] .
$$

As there is an extra-component $\mathcal{E}_{n, m(\mathbf{k}) \|}$ along the direction of $\mathbf{k}_{n, m}, \mathcal{S}_{n, m(\mathbf{k})}$ possess in the TE case an extra-component along the direction of $\mathcal{D}_{n, m(\mathbf{k})}$. The consequence of this extra-component on the group velocity is discussed below.

\subsubsection{Group velocity of the global Bloch wave}

In lossless periodic media, the group velocity $\mathbf{v}_{\mathbf{g}}$ of a Bloch wave is equal to its energy velocity $\mathbf{v}_{\mathbf{e}}$ (Yeh 1979):

$$
\mathbf{v}_{\mathbf{g}}=\mathbf{v}_{\mathbf{e}}=\frac{\left\langle\mathbf{S}_{\mathbf{k}}\right\rangle_{t, s}}{\left\langle\mathbb{E}_{\mathbf{k}}\right\rangle_{t, s}}=\frac{\sum_{n, m} \mathcal{S}_{n, m(\mathbf{k})}}{\frac{1}{2} \mu_{0} H_{0}^{2}}=\sum_{n, m}\left|\mathbf{h}_{n, m(\mathbf{k})}\right|^{2} \mathbf{v}_{n, m(\mathbf{k})},
$$

where $\mathbf{v}_{n, m(\mathbf{k})}$ is the group velocity of $\mathcal{F}_{n, m(\mathbf{k})}$ given by:

$$
\mathbf{v}_{n, m(\mathbf{k})}=\frac{\mathcal{S}_{n, m(\mathbf{k})}}{\mathbb{E}_{n, m(\mathbf{k})}}
$$

According to Equation (30), the group velocity of the global Bloch wave is simply given by the sum of the group velocities $\mathbf{v}_{n, m(\mathbf{k})}$ of the partial electromagnetic plane waves $\mathcal{F}_{n, m(\mathbf{k})}$ weighted by their relative energetic contributions $\left|\mathbf{h}_{n, m(\mathbf{k})}\right|^{2}$. This interesting relation provides an intuitive understanding of the propagation direction of Bloch waves in 2D-PhCs, see Section 4.1. Moreover, this result is valid for both TE and TM polarizations even if the expressions of the group velocities $\mathbf{v}_{n, m(\mathbf{k})}$ are different in each case.

TM polarization:

$$
\mathbf{v}_{n, m(\mathbf{k})}=\frac{\omega}{\left|\mathbf{k}_{n, m}\right|^{2}} \mathbf{k}_{n, m} .
$$

The group velocity $\mathbf{v}_{n, m(\mathbf{k})}$ is simply equal to the phase velocity of the partial electromagnetic TM plane wave $\mathcal{F}_{n, m(\mathbf{k})}$. 
TE polarization:

$$
\mathbf{v}_{n, m(\mathbf{k})}=\frac{\omega}{\left|\mathbf{k}_{n, m}\right|^{2}} \mathbf{k}_{n, m}+\mathbf{v}_{n, m(\mathbf{k}) \perp}
$$

where

$$
\mathbf{v}_{n, m(\mathbf{k}) \perp}=\Re e\left(-\frac{\omega}{\left|\mathbf{k}_{n, m}\right|^{2}} \frac{\mathbf{k}_{n, m} \cdot \mathbf{e}_{n, m(\mathbf{k})}}{\left|\mathbf{h}_{n, m(\mathbf{k})}\right|^{2}} \mathbf{d}_{n, m}^{*}\right) .
$$

The component of $\mathbf{v}_{n, m(\mathbf{k})}$ along the direction of $\mathbf{k}_{n, m}$ is still equal to the phase velocity $\left(\omega /\left|\mathbf{k}_{n, m}\right|^{2}\right) \mathbf{k}_{n, m}$. Nevertheless, as in anisotropic media, the presence of the extra electric component $\mathcal{E}_{n, m(\mathbf{k}) \|}$ leads to a non-zero component $\mathbf{v}_{n, m(\mathbf{k}) \perp}$ of the group velocity along the direction perpendicular to $\mathbf{k}_{n, m}$, see Fig. 2.

Finally, we want to point out that although $\mathbf{k}_{n, m}$ and $\mathbf{v}_{n, m(\mathbf{k})}$ are not parallel, the scalar product $\mathbf{k}_{n, m} \cdot \mathbf{v}_{n, m(\mathbf{k})}=\omega$ is always positive. This shows that all the partial electromagnetic plane waves $\mathcal{F}_{n, m(\mathbf{k})}$ propagate in a standard righthanded material and will be commented on in Section 4.3.

\section{Examples}

In this section, we consider the 2D-PhC structure made of a square lattice of cylindrical air holes etched in a dielectric medium (inset in Fig. 3). The lattice period is ' $\mathrm{a}$ ' and the rod radius is ' $\mathrm{r}$ '. The filling factor $f=\pi \mathrm{r}^{2} / \mathrm{a}^{2}$ is $50 \%$ and the susceptibilities are $\varepsilon_{1}=11$ and $\varepsilon_{2}=1$. The band diagram of this structure is represented in Fig. 3 for each polarization.

\subsection{GRAPHICAL REPRESENTATION OF BLOCH WAVES IN THE RECIPROCAL SPACE}

In order to illustrate graphically the Fourier decomposition of a Bloch wave propagating in the $\mathrm{PhC}$ structure under study, we need to represent on a single diagram all the partial electromagnetic plane waves $\mathcal{F}_{n, m(\mathbf{k})}$ and their respective contributions to the energy density and group velocity.

A possible graphical representation of the partial electromagnetic plane wave $\mathcal{F}_{n, m(\mathbf{k})}$ is given in Fig. 4 where the particular example of $\mathcal{F}_{1,0(\mathbf{k})}$ is considered. Each electromagnetic wave $\mathcal{F}_{n, m(\mathbf{k})}$ can be represented by a disk located at $\mathbf{k}_{n, m}=\mathbf{k}+\mathbf{G}_{n, m}$ : the gray level of the disk indicates its relative energetic contribution $\left|\mathbf{h}_{n, m}(\mathbf{k})\right|^{2}$ to the total Bloch wave (black $\rightarrow 1$, white $\rightarrow 0$ ). From the center of the disk, the vector $\mathbf{v}_{n, m(\mathbf{k})}$ shows its group velocity and 


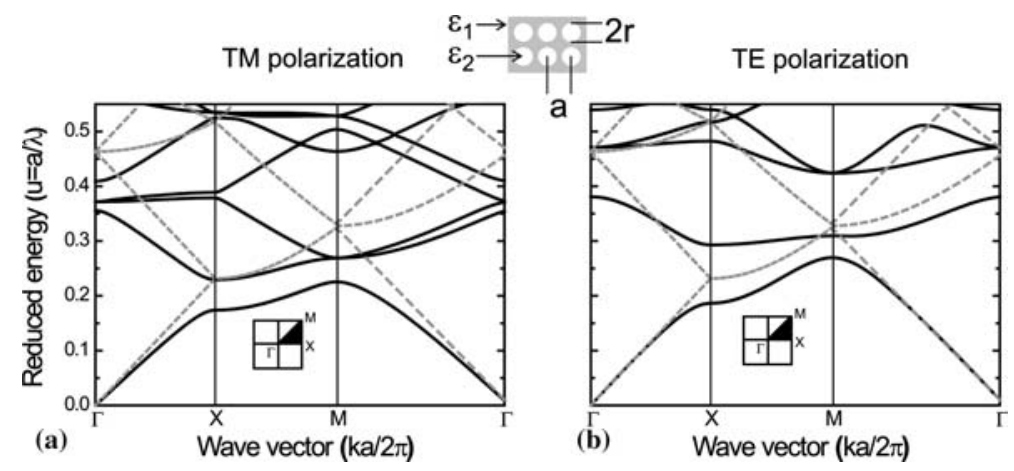

Fig. 3. Photonic band diagrams of the investigated 2D-PhC $\left(\varepsilon_{1}=1\right.$ and $\varepsilon_{2}=11, f=50 \%$, black curve $)$ and of an homogenous medium considered as a PhC with zero modulation $\left(\varepsilon_{1}=\varepsilon_{2}=4.67\right.$, broken gray curve). For comparison, they both have the same average refractive index $\langle n\rangle=2.16$. The three highly symmetric points $\Gamma, \mathrm{M}$ and $\mathrm{X}$ of the square lattice are indicated. The inset shows the $2 \mathrm{D}-\mathrm{PhC}$ structure made of a square lattice of air holes embedded in a dielectric medium. (a) TM polarization (b) TE polarization.

the vector $\left|\mathbf{h}_{n, m(\mathbf{k})}\right|^{2} \mathbf{v}_{n, m(\mathbf{k})}$ its contribution of $\mathcal{F}_{n, m(\mathbf{k})}$ to the global group velocity.

In the TE case, the vectors $\mathbf{k}_{n, m}$ and $\mathbf{v}_{n, m(\mathbf{k})}$ do not necessarily point in the same direction. However, they belong to the same half-space since $\mathbf{k}_{n, m} \cdot \mathbf{v}_{n, m(\mathbf{k})}>0$, see Section 3.2.

We now consider the examples of the TM- and TE-polarized Bloch waves with wave vector $\mathbf{k}=(0.35,0.1)$ propagating in the $2 \mathrm{D}-\mathrm{PhC}$ under study. The eigenvalues problem in Equations (18) and (19) has been solved with $N=10$, and for each polarization, only the solution located in the first transmission band is considered in this section. The corresponding partial electromagnetic plane waves $\left\{\mathcal{F}_{n, m(\mathbf{k})}\right\}$ are represented in Fig. 5a and b with the same representation as in Fig. 4. Here, only the vector $\left|\mathbf{h}_{n, m(\mathbf{k})}\right|^{2} \mathbf{v}_{n, m}$ emerges from the center of each disk in order to show the contribution to the group velocity $\mathbf{v}_{\mathbf{g}}$ of the global Bloch wave. From Equation (30), $\mathbf{v}_{\mathbf{g}}$ is simply given by the vectorial sum of all these vectors. In this particular example, $\mathcal{F}_{0,0(\mathbf{k})}, \mathcal{F}_{-1,0(\mathbf{k})}$, $\mathcal{F}_{0,-1(\mathbf{k})}, \mathcal{F}_{-1,-1(\mathbf{k})}$ are in the first, second, third, fourth Brillouin Zones (BZs), respectively.

We observe in this particular example that the Fourier decomposition of each Bloch wave is strongly dominated by the partial electromagnetic plane wave $\mathcal{F}_{0,0(\mathbf{k})}$ located in the first BZ $\left(\left|\mathbf{h}_{0,0(\mathbf{k})}\right|^{2}>0.92\right)$. The contribution of the other $\mathcal{F}_{n, m(\mathbf{k})}$ is negligible so that the global Bloch wave is very similar to $\mathcal{F}_{0,0(\mathbf{k})}$. According to Equation (30), the group velocity $\mathbf{v}_{\mathbf{g}}$ is therefore very close to the group velocity $\mathbf{v}_{0,0(\mathbf{k})}$ of $\mathcal{F}_{0,0(\mathbf{k})}$. Moreover, we observe in the TE case that the extra-component $\mathbf{v}_{0,0(\mathbf{k}) \perp}$ is very small, so that $\mathbf{v}_{0,0(\mathbf{k})}$ points in a direction very close to $\mathbf{k}_{0,0}$. This result is actually very general: for partial 

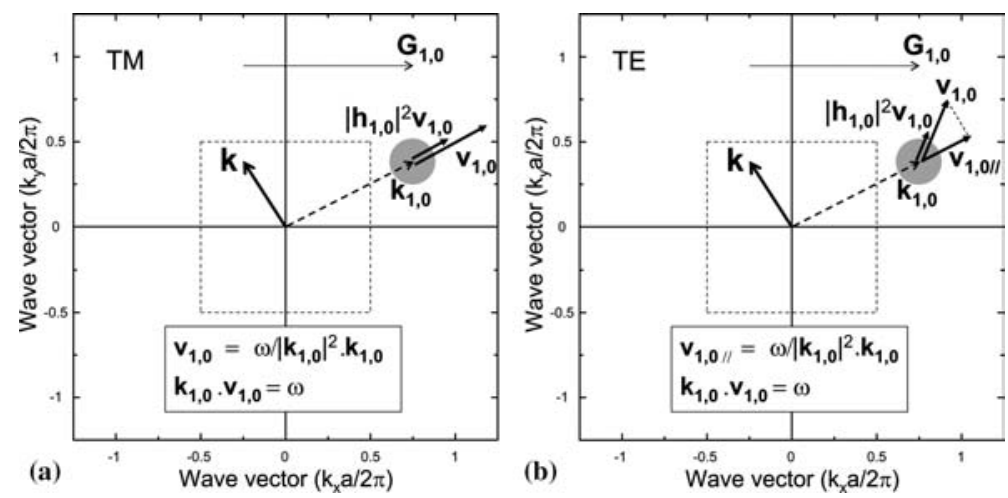

Fig. 4. Graphical representation of the partial electromagnetic plane wave $\mathcal{F}_{1,0(\mathbf{k})}$ in the reciprocal space. The boundaries of the first Brillouin zone are represented by dashed lines. (a) TM polarization (b) TE polarization.

electromagnetic plane waves with high energetic contribution, we have observed that the extra-component $\mathbf{v}_{n, m}(\mathbf{k}) \perp$ of the group velocities is generally small compared to the parallel component $\left(\omega /\left|\mathbf{k}_{n, m}\right|^{2}\right) \mathbf{k}_{n, m}$. This property has been observed in all the cases we have investigated. Nevertheless its theoretical demonstration and domain of validity have not been completely clarified yet.

To conclude, although the decomposition of the group velocity is less straightforward in the TE case, the results are qualitatively similar to the TM case. In particular, when the Fourier decomposition of a Bloch wave is dominated by a single partial electromagnetic plane wave $\mathcal{F}_{n, m(\mathbf{k})}$, the group velocity of the Bloch wave points in a direction very close to $\mathbf{k}_{n, m}$. However, the fact that the Bloch wave always has a dominant component which governs the group velocity has not yet been rigorously demonstrated for the TM case.
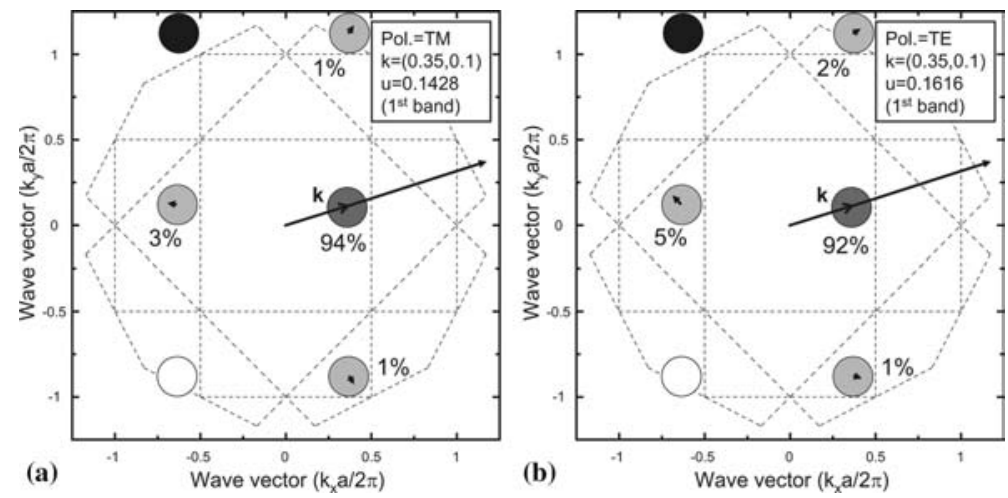

Fig. 5. Graphical representation of the (a) TM- and (b) TE-polarized Bloch waves with the wave vector $\mathbf{k}=(0.35,0.1)$ propagating in the $2 \mathrm{D}-\mathrm{PhC}$ under study (Parameters: $\varepsilon_{1}=1, \varepsilon_{2}=11$ and $f=50$ ). These Bloch waves are located in the first transmission band of the $2 \mathrm{D}-\mathrm{PhC}$ under study. The Brillouin zone boundaries are represented by dashed lines. 


\subsection{CONTINUOUS TRANSITION FROM THE 2D-PHCS TO THE HOMOGENEOUS MEDIUM}

In this section, we consider the TM-polarized Bloch wave with wave vector $\mathbf{k}=(0.35,0.1)$ located in the second transmission band of the $2 \mathrm{D}-\mathrm{PhC}$ described in the introduction of Section 4. Its graphical representation (Fig. 6a) shows that its Fourier decomposition is now strongly dominated by the partial electromagnetic plane wave $\mathcal{F}_{-1,0(\mathbf{k})}$ located in the second BZ $\left(\left|\mathbf{h}_{-1,0(\mathbf{k})}\right|^{2}=0.78\right)$. This example illustrates that care must be taken, when using the common reduced-zone scheme, to identify the dominant wave vector of the Bloch wave. Indeed, it would be erroneous to assume that the dominant wave vector is always the wave vector $\mathbf{k}=\mathbf{k}_{0,0}$ of the first BZ. Here, the partial electromagnetic plane wave $\mathcal{F}_{0,0(\mathbf{k})}$ has for example just a negligible energetic contribution $\left(\left|\mathbf{h}_{0,0(\mathbf{k})}\right|^{2}<0.01\right)$ and has therefore just a negligible influence on the properties of the global Bloch wave.

Problems resulting from an analysis focused on the first BZ are discussed in details in (Lombardet et al. 2005). As an illustration, let us reduce continuously the index contrast of the 2D-PhC to zero: the homogeneous medium is now described as a $2 \mathrm{D}-\mathrm{PhC}$ with zero index contrast. After band folding, the electromagnetic plane wave represented in Fig. $6 \mathrm{~b}$ is characterized by the wave vector $\mathbf{k}=(0.35,0.1)$ whereas only the wave vector $\mathbf{k}_{-1,0}=(-0.65,0.1)$ has a genuine physical meaning for this wave.

\subsection{ARE PHCS LEFT-HANDED MATERIALS IN THE SECOND TRANSMISSION BAND?}

Left-handed materials (LHMs) are generally characterized by the negative sign of the scalar product $\mathbf{k} \cdot \mathbf{v}_{\mathbf{g}}$, where $\mathbf{k}$ is the wave vector and $\mathbf{v}_{\mathbf{g}}$ the group
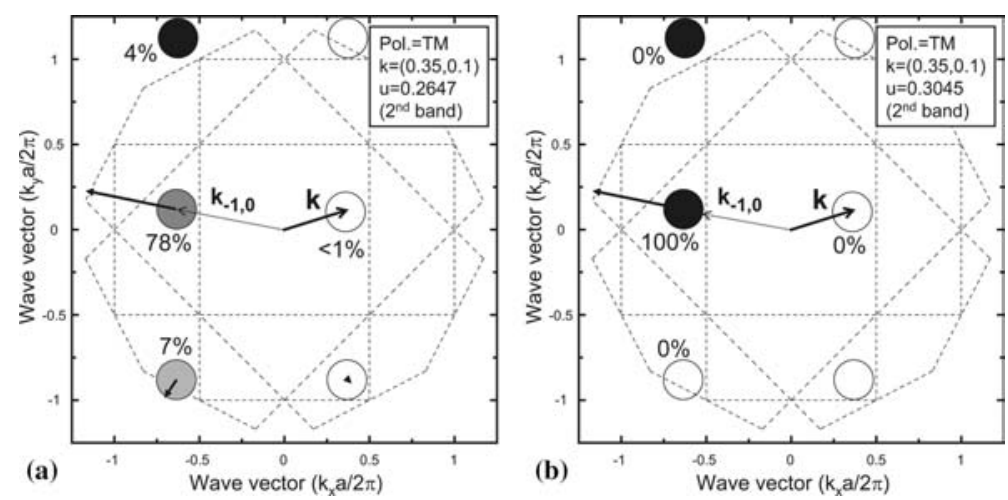

Fig. 6. Graphical representation of the TM-polarized Bloch wave with the wave vector $\mathbf{k}=(0.35,0.1)$ and located in the second transmission band of (a) the 2D-PhC under study and (b) the homogeneous medium described in Fig. 3. 
velocity (Veselago 1968). In order to calculate this scalar product for a Bloch wave, a single wave vector ' $k$ ' must be assigned to the considered Bloch wave. The wave vector $\mathbf{k}=\mathbf{k}_{0,0}$ of the first BZ is generally chosen. In the second transmission band, this scalar product is negative, thus suggesting that $\mathrm{PhCs}$ behave as LHMs in this frequency range.

However, the Fourier decomposition of Bloch waves clearly shows that these waves cannot be unambiguously characterized by a single wave vector. Thus, the physical meaning of the scalar product ' $\mathbf{k}$ ' $\mathbf{v}_{\mathbf{g}}$ is not clear for a Bloch wave: as a matter of fact, this scalar product is negative for the wave vector $' \mathbf{k}$ ' $=\mathbf{k}_{0,0}$ located in the first $\mathrm{BZ}$, but positive for the wave vector ' $\mathbf{k}$ ' $=\mathbf{k}_{-1,0}$ of the dominant partial electromagnetic plane wave. The negative sign of the product $\mathbf{k} . \mathbf{v}_{\mathbf{g}}=\mathbf{k}_{0,0} \cdot \mathbf{v}_{\mathbf{g}}$ reported in previous papers has limited physical meaning for optical Bloch waves. Therefore, it should not be invoked to characterize the LHM properties of the second transmission band of 2D-PhCs.

\section{Conclusion}

We have demonstrated that an optical Bloch wave with the wave vector $\mathbf{k}$ can be decomposed as series of partial electromagnetic plane waves $\left\{\mathcal{F}_{n, m(\mathbf{k})}\right\}$. Each of these waves satisfies Maxwell's equations and is characterized by the wave vector $\mathbf{k}_{n, m}=\mathbf{k}+\mathbf{G}_{n, m}$, where $\mathbf{G}_{n, m}$ is a vector of the reciprocal lattice. In the TM case, $\mathcal{F}_{n, m(\mathbf{k})}$ is identical to an electromagnetic plane wave propagating in an homogenous medium with the permittivity $\varepsilon_{0}\left|\mathbf{k}_{n, m} c / \omega\right|^{2}$. In the TE case, the electric field of $\mathcal{F}_{n, m(\mathbf{k})}$ possess an extra-component along the direction of $\mathbf{k}_{n, m}$. Even if this component does not result from anisotropic effects, the properties of $\mathcal{F}_{n, m(\mathbf{k})}$ are very similar to those of an electromagnetic plane wave propagating in an anisotropic medium. In both cases, the global Bloch wave is characterized by the relative amplitudes of the different $\mathcal{F}_{n, m(\mathbf{k})}$ which are imposed by Maxwell's equations. The energy density of the global Bloch can be decomposed as the sum of the energies densities of the different $\mathcal{F}_{n, m(\mathbf{k})}$. Its group velocity is given by the sum of the group velocities of the $\mathcal{F}_{n, m(\mathbf{k})}$ weighted by their relative energetic contribution. This decomposition provides a simple and intuitive method for understanding and investigating the propagative and refractive properties of optical Bloch waves in 2D-PhCs.

\section{Acknowledgements}

The authors would like to acknowledge P.S.J. Russell for very helpful discussions. This work was supported by the Swiss National Science 
Foundation in the framework of the research initiative NCCR Quantum Photonics and by IST project PCIC (Contract No. 1999-11239).

\section{References}

Baba, T. and M. Nakamura. IEEE J. Quant. Electron. 38(7) 909, 2002.

Chu, R.S. and T. Tamir. Proc. IEEE Trans. MTT 18(6) 486, 1970.

Chu, R.S. and T. Tamir. Proc. IEE London 119(7) 797, 1972.

Cubukcu, E., K. Aydin, E. Ozbay, S. Foteinopolou and C.M. Soukoulis. Phys. Rev. Lett. 91(20) 207401, 2003.

Foteinopoulou, S., E.N. Economou and C.M. Soukoulis. Phys. Rev. Lett. 90(10) 107402, 2003.

Kosaka, H. T. Kawashima, A. Tomita, M. Notomi, T. Tamamura, T. Sato and S. Kawakami. Phys. Rev. B 58(16) 10096, 1998.

Kosaka, H. T. Kawashima, A. Tomita, M. Notomi, T. Tamamura, T. Sato and S. Kawakami. Appl. Phys. Lett. 74(9) 1212, 1999.

Lombardet, B., L. Dunbar, R. Ferrini and R. Houdré. 2005 Accepted for publication in J. Optical Soc. Am.

Luo, C., and S.G. Johnson and J.D. Joannopoulos and J.B. Pendry. Phys. Rev. B 65(20) 201104, 2002.

Notomi, M. Phys. Rev. B 62(16) 10696, 2000.

Parimi, P.V., W.T.T. Lu, P. Vodo and S. Sridhar. Nature 426(6965) 404, 2003.

Plihal, M. and A.A. Maradudin. Phys. Rev. B 44(16) 8565, 1991.

Russell, P.S.J. Phys. Rev. Lett. 56(6) 596, 1986.

Russell, P.S.J. Appl. Phys. B - Photophys. Laser Chem. 39(4) 231, 1986.

Sakoda K. Optical Properties of Photonic Crystals. Springer-Verlag, Berlin, Germany, 2001.

Veselago, V.G., Soviet Phys. Uspekhi Ussr 10(4) 509, 1968.

Witzens, J., M. Loncar and A. Scherer. IEEE J. Selected Topics Quantum Electron. 8(6) 1246, 2002.

Witzens, J. and A. Scherer. J. Optical Soc. Am. A 20(5) 935, 2003.

Wu, L.J., M. Mazilu, T. Karle and T.F. Krauss. IEEE J. Quantum Electron. 38(7) 915, 2002.

Yeh, P. J. Optical Soc. Am. 69 (5) 742, 1979.

Zengerle, R. J. Modern Optics 34 (12) 1589, 1987. 